\title{
PREDICTION FOR IRREGULAR OCEAN WAVE AND FLOATING BODY MOTION BY REGULARIZATION: PART 1. IRREGULAR WAVE PREDICTION
}

\begin{abstract}
Summary
Ocean waves can be explained in terms of many factors, including wave spectrum, which has the characteristics of wave height and periodicity, directional spreading function, which has a directional property, and random phase, which randomly represents a certain property. Under the assumption of a linear system, ocean waves show irregular behaviours, which can be observed in the forms of wave spectrum, directional spreading function, and complex phase calculations using the method of linear superposition. Ocean waves, which include a variety of periodic elements, exhibit direct proportionality between their period and propagation velocity. The purpose of this study was to understand the phase components of the period and to make exact calculations on the deterministic phase in order to make predictions on ocean waves. However, measurements of actual ocean waves exist only in the form of information on wave elevation, so we faced an inverse problem of having to analyse this information and calculate the deterministic phase. Regularization was used as part of the solution, and various methods were used to obtain stable values.
\end{abstract}

Key words: $\quad$ deterministic phase, ocean wave, prediction, regularization, L-curve.

\section{Introduction}

Recent trends show an increase in the construction of floating offshore structures capable of deep sea operations and offshore support vessels (OSVs) that can be used under extreme conditions to mine natural resources in the sea. The structural and dynamic stabilities of such floating bodies (offshore structures and vessels) are affected by components such as sea surface winds, surface currents, and ocean waves, which act as external forces. In particular, ocean waves are closely related to the dynamic performance of floating bodies, and studies on the motion of floating bodies and induced ocean waves are being conducted continuously. In addition, the continual operation of offshore plants under extreme conditions is directly connected to mining resources, leading to profitability. However, casualty reduction in foul weather is a key variable in evaluating a system's reliability, necessitating research on the motion of floating bodies and induced ocean waves with its prediction.

Traditionally, the measurement of ocean waves is based on point measurement using insitu types of buoys and acoustic sensors. Measurements and analyses at a particular point 
were used to calculate statistical properties - wave height and wave period - of the corresponding sea area. Meanwhile, due to the need for wide-ranged, macroscopic research on ocean waves, remote sensing methods that used a radar or a satellite have been on the rise, aside from existing in-situ measurement tools. The remote sensing method has the advantage of being able to overcome the limitations of the in-situ point-measurement method. Representative studies on ocean wave analysis using $\mathrm{x}$-band radar include research on wave direction calculation via the three-dimensional Fourier analysis of incoherent $\mathrm{x}$-band marine radar images and ambiguity in wave directionality - distinguishing incoming from outgoing waves (Young and Rosenthal [1], Atanassov et al. [2]). The above study is on wave directionality analysis, and the wave height estimation through a marine radar image analysis was first applied in Ziemer and Günther [3]. In addition, there has been work done on converting the reflective intensity of electromagnetic waves generated by an x-band radar into a wave elevation map (Nieto Borge et al. [4]).

There have been recent studies on wave prediction using wave elevation maps measured by an x-band radar (Blondel and Naaijen [5], Blondel and Naaijen [6]). Generally, in the case of wave elevation maps using the $\mathrm{x}$-band radar, it is possible to measure an area covered by a radius of 1 to $3 \mathrm{~km}$ from the point of measurement. In addition, due to the dispersive characteristic of the ocean wave - phase velocity is directly proportional to wave length - the measured wave elevation does not accurately reach the floating body. It is for this reason that an algorithm for ocean wave prediction is needed, and there are several similar studies (Naaijen and Hujismans [7], Clauss et al. [8], Naaijen et al. [9], Wu [10], Blondel [11]). While the above theses share a commonality of calculating the wave phase to predict ship motion, Clauss et al. [8] and Kosleck [12] calculate the phases of the real and imaginary parts using the FFT on the wave elevation map, and Naaijen and Hujismans [13] use singular value decomposition to calculate the phases of waves. Wave phase data obtained in this manner are used, along with the RAO values previously computed for each vessel mode, to predict vessel motion in time series according to waves that are going to reach the vessel.

In Kwon et al. [14], the Gaussian wave packet was treated as a wave amplitude spectrum, and the integrand to which linear superposition was applied was evaluated analytically. In addition, after having carried out the inverse matrix calculations, final results were obtained by regularization in order to solve the ill-conditioned problem. In Blondel et al. [15], verifications were done by assuming a model that predicted two-dimensional irregular waves using the High-Order Spectral method, and by taking gauge measurement data into consideration. In particular, non-linear waves could be predicted by extending the existing second-order method to a third-order method.

Additionally, theoretical research on accuracy and experimental validation for the prediction of irregular wave were conducted by exploiting a directional hybrid wave model (Zhang et al. [16] and Zhang et al. [17]). Belmont et al. [18] and Guang et al. [19] forecasted irregular wave with the calculation of deterministic phase through filter.

In part 1, this paper proposes a wave prediction algorithm essential to technologies previously mentioned. Wave spectra are calculated based on the Fourier analysis on wave elevations measured at a particular point, and deterministic phases are calculated intuitively through singular value decomposition. In particular, when calculating the deterministic phase, wave elevation data, which are obtained from a wave gauge in a limited manner, may be considered an ill-posed problem with instability due to the lack of measured values and measurement errors. Therefore, the Tikhonov regularization method is used to address the instability of this inverse problem, and the L-curve method is introduced to evaluate an appropriate regularization coefficient. 


\section{Deterministic phase: "the key to irregular wave"}

Ocean waves are generated by several factors including gravitational acceleration, wind, and current, and it is difficult to clearly investigate and accurately reproduce the physical causes. Nonetheless, we conducted a study on irregular ocean waves using deterministic algorithms with statistical foundations from the past. One representative method is the realization of irregular ocean waves using wave spectra and random phases.

These irregular waves can be expressed as a sum of linear waves by linear superposition. First, the sinusoidal progressing linear wave is as follows:

$$
\eta(x, t)=\mathbb{R}\left\{A e^{-i(k x-\omega t)}\right\}
$$

where $A$ is the wave amplitude, $k$ is the wave number, $x$ is the location information, $\omega$ is the wave frequency, and $t$ is time.

A combination of the unit amplitude $A$ and the discrete number of the sinusoidal wave were used to express irregular waves, and the irregular elevation with a random phase at a fixed position is as follows:

$$
\eta(t)=\mathbb{R}\left\{\sum_{p=1}^{M} A_{p} e^{i\left(\omega_{p} t+\varphi_{p}\right)}\right\}
$$

where $M$ is the number of frequency components of irregular waves. An example of unit amplitude irregular waves using eq. (2) is shown in Fig. 1. In Fig. 1, a total of eight regular wave components for $\omega=\{0.314, \cdots, 1.047\}$ were used, and the random phase $\varphi_{p}$ had eight components as well, in the range of $0 \leq \varphi_{p}<2 \pi$.

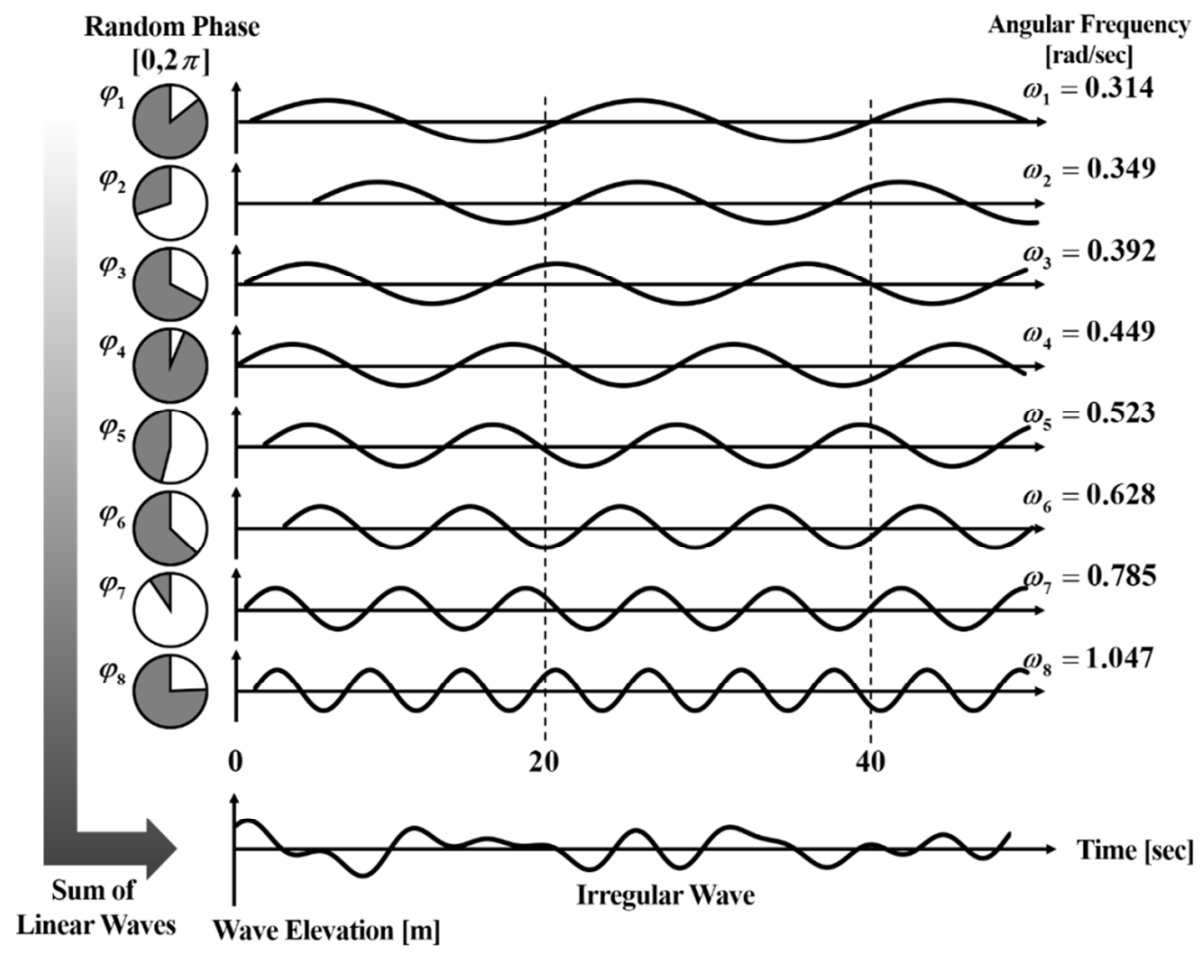

Fig. 1 Example of unit amplitude irregular wave with deterministic phase 
The amplitude spectrum introduced for the application of the wave spectrum in eq. (3) is:

$$
A_{p}=\sqrt{2 S\left(\omega_{p}\right) \Delta \omega}
$$

The empirical wave spectrum, $S\left(\omega_{p}\right)$, was used to reflect the characteristics of ocean waves, and in this paper, the modified two-parameter JONSWAP spectrum was used, see Kim $[20]$.

\section{Decomposition of irregular wave}

In the study by Janssen et al. [21], also related to wave prediction, singular value decomposition is used to calculate the entire phase to confirm the potential of wave prediction. An identical method was used for wave prediction in Naaijen et al. [9] and Naaijen and Hujismans [13], but no evaluation was made on the instability that could be caused by the use of singular value decomposition. This instability can be caused by the lack of values measured by the wave gauge and measurement errors, and the ultimate solution can be physically meaningless or numerical calculations may not converge. In a similar study presented by Kwon et al. [14], in which the condition number of the singular value matrix is confirmed in order to solve the ill-posed problem, and Tikhonov regularization is used to reach a solution. However, it is not a method that directly produces phase by setting the spectrum as a variable, but has the feature of using the Gaussian wave packet on the input spectrum and directly comparing the form of input versus output wave spectra.

\subsection{Matrix form of two dimensional wave}

In this paper, the prediction algorithm has the advantage of making intuitive calculations by treating the random phase $\varphi_{p}$ as a variable and using only the wave elevation at a fixed point. To avoid confusion in terminology, we define the variable-treated random phase in the inverse problem as "deterministic phase." The deterministic phase is an important factor that influences the elevation of irregular waves when making superposition calculations on linear waves of various periods. Particularly, in the case of the complex form in eq. (2), the summation inside the exponent can be transformed into the form of multiplication of exponents. In other words, it can be expressed as the multiplication of the terms temporal information, $\omega_{p} t$, and the deterministic phase, $\varphi_{p}$, as shown below:

$$
\left[\begin{array}{c}
\eta\left(t_{1}\right) \\
\eta\left(t_{1}\right) \\
\vdots \\
\eta\left(t_{N}\right)
\end{array}\right]=\left[\begin{array}{cccc}
A_{1} e^{i \omega_{1} t_{1}} & A_{2} e^{i \omega_{2} t_{1}} & \ldots & A_{M} \cdot e^{i \omega_{M}, t_{1}} \\
A_{1} e^{i \omega_{1} t_{2}} & A_{2} e^{i \omega_{2} t_{2}} & \cdots & A_{M} \cdot e^{i \omega_{M}, t_{2}} \\
\vdots & \vdots & \ddots & \vdots \\
A_{1} e^{i \omega_{1} t_{N}} & A_{2} e^{i \omega_{2} t_{N}} & \cdots & A_{M} \cdot e^{i \omega_{M}, t_{N}}
\end{array}\right]\left[\begin{array}{c}
e^{i \varphi_{1}} \\
e^{i \varphi_{2}} \\
\vdots \\
e^{i \varphi_{M^{\prime}}}
\end{array}\right]
$$

The first matrix on the right-hand side of eq. (4) can be expressed as:

$$
\left[\begin{array}{c}
\eta\left(t_{1}\right) \\
\eta\left(t_{1}\right) \\
\vdots \\
\eta\left(t_{N}\right)
\end{array}\right]=\left[\begin{array}{cccc}
e^{i \omega_{1} t_{1}} & e^{i \omega_{2} t_{1}} & \ldots & e^{i \omega_{M}, t_{1}} \\
e^{i \omega_{1} t_{2}} & e^{i \omega_{2} t_{2}} & \cdots & e^{i \omega_{M}, t_{2}} \\
\vdots & \vdots & \ddots & \vdots \\
e^{i \omega_{1} t_{N}} & e^{i \omega_{2} t_{N}} & \cdots & e^{i \omega_{M} t_{N}}
\end{array}\right]\left[\begin{array}{cccc}
A\left(\omega_{1}\right) & 0 & \cdots & 0 \\
0 & A\left(\omega_{2}\right) & \cdots & 0 \\
\vdots & \vdots & \ddots & \vdots \\
0 & 0 & \cdots & A\left(\omega_{M^{\prime}}\right)
\end{array}\right]\left[\begin{array}{c}
e^{i \varphi_{1}} \\
e^{i \varphi_{2}} \\
\vdots \\
e^{i \varphi_{M^{\prime}}}
\end{array}\right] .
$$


In other words,

$$
Y=T A P \text {. }
$$

The left-hand side of the equation, $Y$, is the number of elevations measured by the wave gauge. For instance, making measurements for $5 \mathrm{~s}$ with a wave gauge with a sampling frequency set to $100 \mathrm{~Hz}$ would yield $N=500$. Therefore, it is an $[N, 1]$ matrix. The first matrix on the right-hand side, $T$, represents temporal information and has the dimensions of $\left[N, M^{\prime}\right]$. For the wave prediction problem in this study, only the data on wave elevation, $\eta$, was measured by the wave gauge, so $M^{\prime}$ is set arbitrarily. The second matrix on the righthand side of the equation, $A$, represents the amplitude spectrum, and can be calculated by the spectral analysis of the measured wave elevation. It is a diagonal matrix with the dimensions of $\left[M^{\prime}, M^{\prime}\right]$. As for the final matrix on the right-hand side, $P$, it is treated as the deterministic phase, which is a variable needed in this paper, and has the dimensions of $\left[M^{\prime}, 1\right]$.

Therefore, a summary of each variable is as follows:

a. The left-hand side, $Y$, is the wave elevation data collected by the wave gauge at a fixed point, with the dimensions of $[N, 1]$.

b. $T$ on the right-hand side is temporal information, composed of temporal data measured by the wave gauge and an arbitrary number of $\omega$ components, with the dimensions of $\left[N, M^{\prime}\right]$.

c. $A$ on the right-hand side is the amplitude spectrum, calculated by the spectral analysis on the wave elevation measured for $Y$ on the left-hand side. It is composed of the number of $\omega$ components, as in the diagonal matrix, with the dimensions of $\left[M^{\prime}, M^{\prime}\right]$.

d. $P$ on the right-hand side is the deterministic phase, treated as a variable, and is composed of the same number of $\omega$ components as in one dimensional matrix (vector), with the dimensions of $\left[M^{\prime}, 1\right]$.

\section{Calculation of deterministic phase}

\subsection{Singular value decomposition}

Single value decomposition is a method of matrix diagonalization, where the variable matrix $T$ of the dimensions $\left[N, M^{\prime}\right]$ in eq. (6) is defined as:

$$
T_{N M^{\prime}}=Z_{N N^{S}} \Omega_{N M^{\prime}} V_{M^{\prime} M^{\prime}}{ }^{T}
$$

where

$$
Z Z^{T}=I, V V^{T}=I, \Omega=\operatorname{diag}\left(\lambda^{1 / 2}\right)
$$




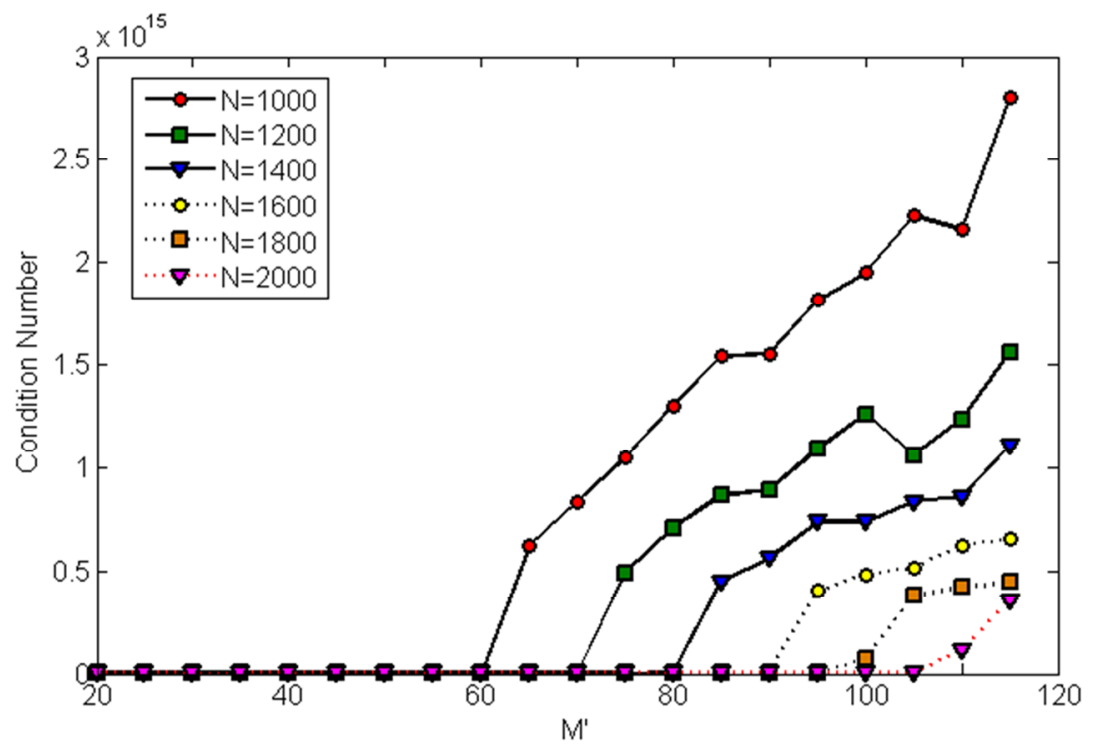

Fig. 2 Example of condition number of $\Omega$ as variable temporal segment

\subsection{Condition number for ill-conditioned system}

In ill-conditioned systems, even small variations in the coefficients can cause great changes in the desired solution. In this case, the condition number of the matrix coefficient must be verified. In particular, matrix $T$ is composed of the time component $t$ and the frequency component $\omega$, necessitating the calculation of the condition number according to the change in the magnitudes of the two components. The optimal solution can thus be found by forming a well-conditioned matrix. Matrix $\Omega$ is a diagonal matrix whose diagonal elements are the square roots of the eigenvalues, and its condition number is:

$$
\text { Condition Number of } \Omega=\|[\Omega]\|\left\|[\Omega]^{-1}\right\| \text {. }
$$

Figure 2 is an example of the condition number of $\Omega$, where the $x$-axis is $M^{\prime}$, the number of frequency components, and shows the change in the condition number for the six temporal segment numbers $N$. As the condition number increases, the matrix becomes illconditioned, increasing the error in the desired solution. Particularly in Fig. 2, as the temporal segment number $N$ increases, the frequency segment number $M^{\prime}$ increases as well, where the condition number is small. This shows a tendency of generating a well-conditioned matrix.

\subsection{Spectral analysis of matrix $A$}

Matrix $Y$, which is measured with the wave gauge, represents the wave elevation data in terms of time and can be transformed into the frequency domain through the Fourier analysis. To minimize noise in the measured signal, Welch's Power Spectral Density [22] was used:

$$
S_{e}(\omega)=\frac{1}{N_{w} T} \sum_{j=1}^{N_{w}}\left|Y_{j}(\omega)\right|^{2}
$$

where $N_{w}$ is called the "windowing size" or "block size," representing the number of pieces it is split into. $Y_{j}(\omega)$ is the $\mathrm{j}^{\text {th }}$ split FFT result. 


\subsection{Regularization with L-curve}

The Tikhonov regularization method has been used to overcome the instability of the ill-posed inverse problem. This method solves the problem of instability by adding the positive-definite regularized function to the error function and minimizing the following function (Johnston and Gulrajani [23]):

$$
\min (P)=\|T A P-Y\|^{2}+\alpha\|P\|^{2}
$$

where $P$ is the deterministic phase matrix, treated as a solution matrix, and matrices $T$ and $A$ are calculated in previous steps. \|\| is the Euclidean norm, and $\alpha$ is the regularization parameter, which determines the residual error in the first term on the right-hand side and the weight of the solution norm in the second term. In other words, when the regularization method is applied, as $\alpha$ increases, the solution may stabilize, but when it is too large, it is difficult to obtain an exact solution. Therefore, an appropriate control over the $\alpha$ value is important in stabilizing the solution and finding an accurate solution. Substituting eq. (6) and eq. (7) into eq. (11) yield the following:

$$
P(\alpha)=\left\{(T A)^{T}(T A)+\alpha I\right\}^{-1}(T A)^{T} Y .
$$

Methods that determine the coefficients in this kind of linearized problems include the L-Curve Method and the Generalized Cross Validation. In this research, the regularization coefficient was determined by the L-Curve Method and is as follows (Johnston and Gulrajani [23]):

$$
\zeta(\alpha)=\log \|T A P-Y\|
$$

and

$$
\xi(\alpha)=\log \|P\|
$$

Substituting eq. (12) into eq. (13) and eq. (14):

$$
\zeta(\alpha)=\log \left\|T A\left\{(T A)^{T}(T A)+\alpha I\right\}^{-1}(T A)^{T} Y-Y\right\|
$$

and

$$
\xi(\alpha)=\log \left\|\left\{(T A)^{T}(T A)+\alpha I\right\}^{-1}(T A)^{T} Y\right\| .
$$

Figure 3 is an example of the L-Curve approximation, where the $\mathrm{x}$-axis is $\zeta(\alpha)$ and the $y$-axis is $\xi(\alpha)$. The corner value is at $\alpha=0.039893$. The final deterministic phase matrix $P$ can be obtained by substituting the $\alpha$ value calculated from the L-Curve approximation into eq. (12). 


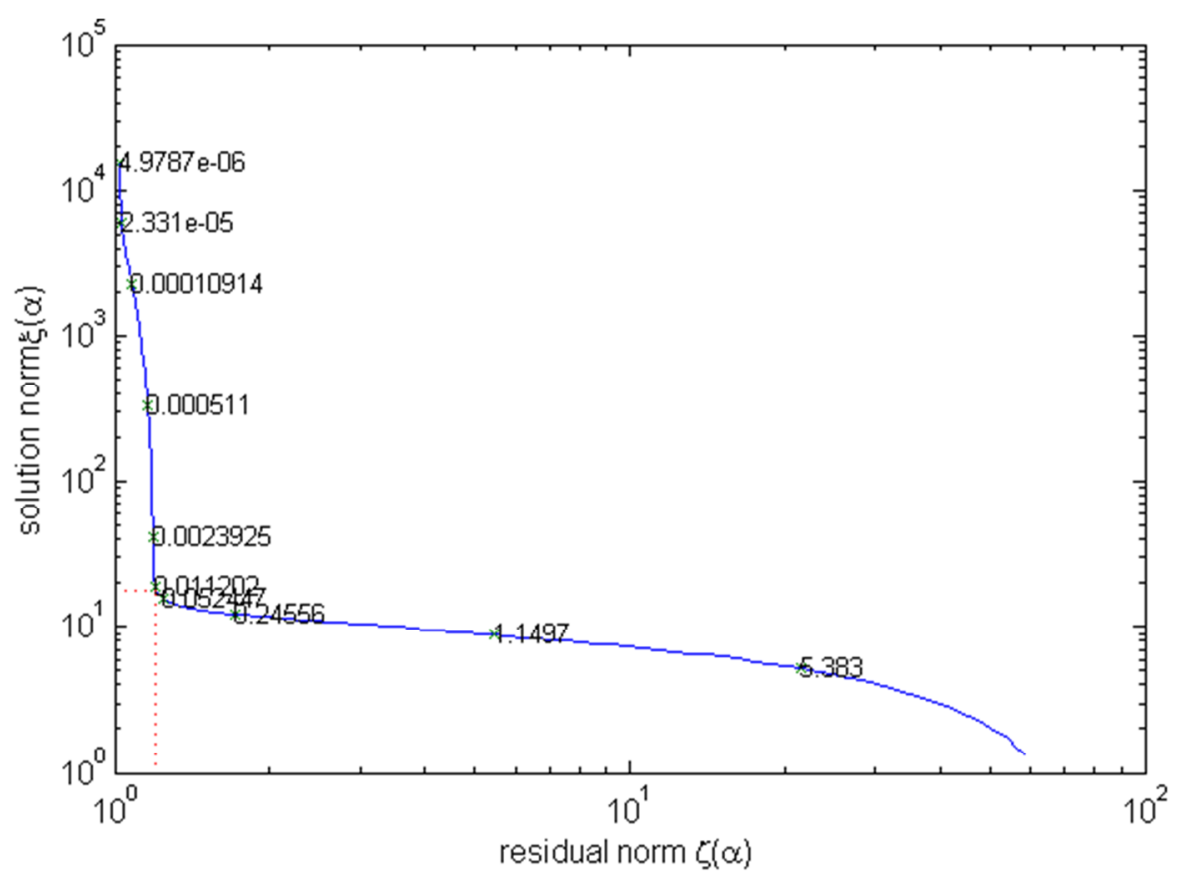

Fig. 3 Example of L-Curve approximation

4.5 Wave prediction using calculated deterministic phase

To predict irregular waves at a particular point using the deterministic phase matrix $P$ obtained before, spatial information must be considered. In the case of the temporal matrix $T$ in eq. (4) to eq. (6) mentioned previously, its value at $x=x_{p}$ may be considered, so we propose the following spatiotemporal matrix $T_{S}$ :

$$
T_{S}=\left[\begin{array}{cccc}
e^{-i\left(k_{1} x_{p}-\omega_{1} t_{1}\right)} & e^{-i\left(k_{2} x_{p}-\omega_{2} t_{1}\right)} & \ldots & e^{-i\left(k_{M}, x_{p}-\omega_{M}, t_{1}\right)} \\
e^{-i\left(k_{1} x_{p}-\omega_{1} t_{2}\right)} & e^{-i\left(k_{2} x_{p}-\omega_{2} t_{2}\right)} & \cdots & e^{-i\left(k_{M}, x_{p}-\omega_{M}, t_{2}\right)} \\
\vdots & \vdots & \ddots & \vdots \\
e^{-i\left(k_{1} x_{p}-\omega_{1} t_{N}\right)} & e^{-i\left(k_{2} x_{p}-\omega_{2} t_{N}\right)} & \cdots & e^{-i\left(k_{M}, x_{p}-\omega_{M}, t_{N}\right)}
\end{array}\right] .
$$

The final predicted irregular wave is:

$$
\begin{array}{r}
{\left[\begin{array}{c}
\eta\left(t_{1}\right) \\
\eta\left(t_{1}\right) \\
\vdots \\
\eta\left(t_{N}\right)
\end{array}\right]=\left[\begin{array}{cccc}
e^{-i\left(k_{1} x_{p}-\omega_{1} t_{1}\right)} & e^{-i\left(k_{2} x_{p}-\omega_{2} t_{1}\right)} & \ldots & e^{-i\left(k_{M}, x_{p}-\omega_{M}, t_{1}\right)} \\
e^{-i\left(k_{1} x_{p}-\omega_{1} t_{2}\right)} & e^{-i\left(k_{2} x_{p}-\omega_{2} t_{2}\right)} & \cdots & e^{-i\left(k_{M^{\prime}} x_{p}-\omega_{M}, t_{2}\right)} \\
\vdots & \vdots & \ddots & \vdots \\
e^{-i\left(k_{1} x_{p}-\omega_{1} t_{N}\right)} & e^{-i\left(k_{2} x_{p}-\omega_{2} t_{N}\right)} & \cdots & e^{-i\left(k_{M^{\prime}} x_{p}-\omega_{M^{\prime}}, t_{N}\right)}
\end{array}\right]} \\
\\
{\left[\begin{array}{cccc}
A\left(\omega_{1}\right) & 0 & \cdots & 0 \\
0 & A\left(\omega_{2}\right) & \cdots & 0 \\
\vdots & \vdots & \ddots & \vdots \\
0 & 0 & \cdots & A\left(\omega_{M^{\prime}}\right)
\end{array}\right]\left[\begin{array}{c}
e^{i \varphi_{1}} \\
e^{i \varphi_{2}} \\
\vdots \\
e^{i \varphi_{M^{\prime}}}
\end{array}\right] .}
\end{array}
$$


In other words,

$$
Y=T_{S} A P .
$$

As for the amplitude spectrum matrix $A$ and the deterministic phase matrix $P$, taking note of their similar nature regardless of temporal and spatial information, the earlier step calculations were used again under the assumption of harmonic oscillation wave. However, we used just the spatiotemporal matrix $T_{S}$, to which spatial information was added, to formulate eq. (19) so that irregular waves could be predicted at a desired point.

\subsection{Predictable zone}

The speed of an ocean wave is directly proportional to the wavelength due to the dispersive property of an ocean wave. Using this property, the maximum and minimum wave frequency values from the wave spectrum energy density were determined, and the group velocity was calculated to determine the measureable region of a wave. Figure 4 presents an explanation of the predictable area, based on the irregular wave measured at a particular point. The spectrum energy density was set to $90 \%$ for the yellow area, and that set to $95 \%$ was the grey area.

Using the linear dispersion relation and the Newton-Raphson method on $\omega_{\text {Min }}$ and $\omega_{\operatorname{Max}}$ values given for $k_{\operatorname{Min}}$ and $k_{\operatorname{Max}}$ calculations, $C_{g M i n}$ and $C_{g M a x}$ can be calculated from eq. (20):

$$
C_{g}=\frac{1}{2} \sqrt{\frac{g}{k} \tanh k h}\left(1+\frac{2 k h}{\sinh 2 k h}\right)
$$

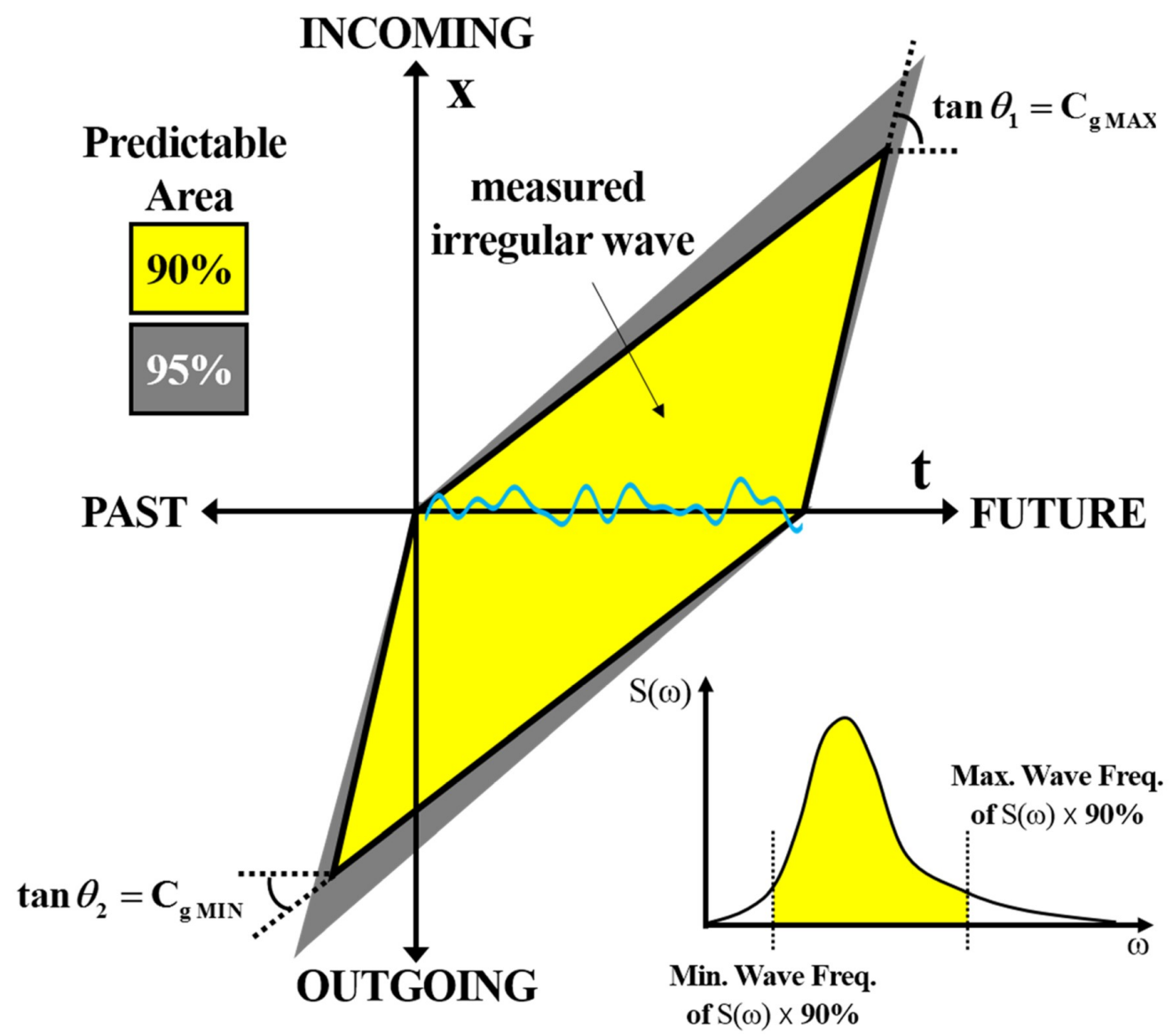

Fig. 4 Predictable zone of irregular wave 


\section{Result}

To draw the wave prediction results, the study was performed under the following conditions:

a. Calculate the deterministic phase by using only the information, $\eta(0, t)$, on wave elevation in the time domain at $x=0$ and restructure the wave elevation using this data. Because it is essential to verify the validity of the deterministic phase, do so by using regular waves for the unit amplitude.

b. Generate irregular waves at one point and calculate the deterministic phase using the inverse method. Finally, make predictions on irregular waves at various points. When doing so, set a predictable region, as shown in Fig. 4, in order to increase accuracy.

5.1 Regular wave and its inversion using regularization method

Figure 5 presents the results obtained for the corner value of the L-Curve in the inverse problem using regular wave elevation. The L-Curve plays an essential role in regularization, and if the value is too great or too small, the value of the deterministic phase, an unknown quantity, has lower precision. This relationship is directly linked to the accuracy of predictions and inverted wave elevations, so it is very important to find the optimum value for the regularization and the L-Curve.

In this problem, the deterministic phase was calculated using corner values obtained from the L-Curve, and the regular waves were restructured, whose results are shown in Fig. 6. The results show an exact match between the generated regular waves and wave elevations restructured by the calculations of the deterministic phase.

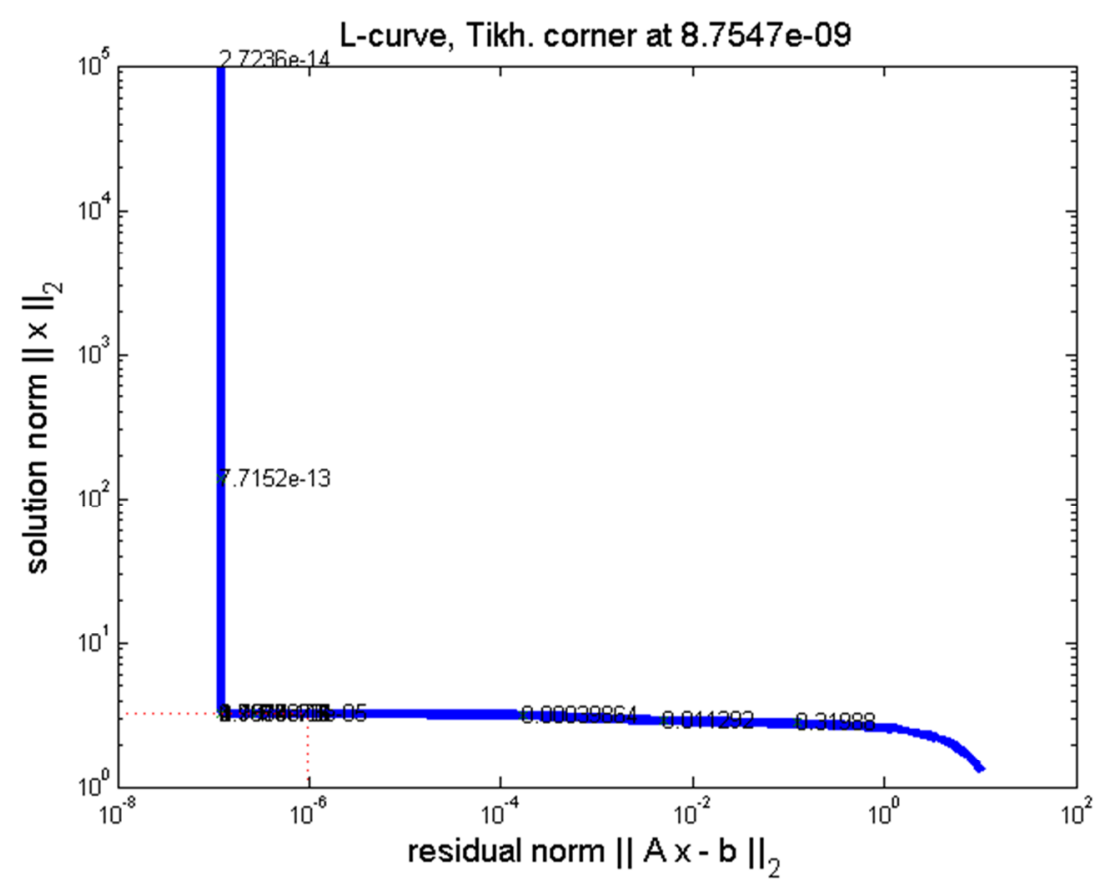

Fig. 5 Result of L-Curve for regular wave 


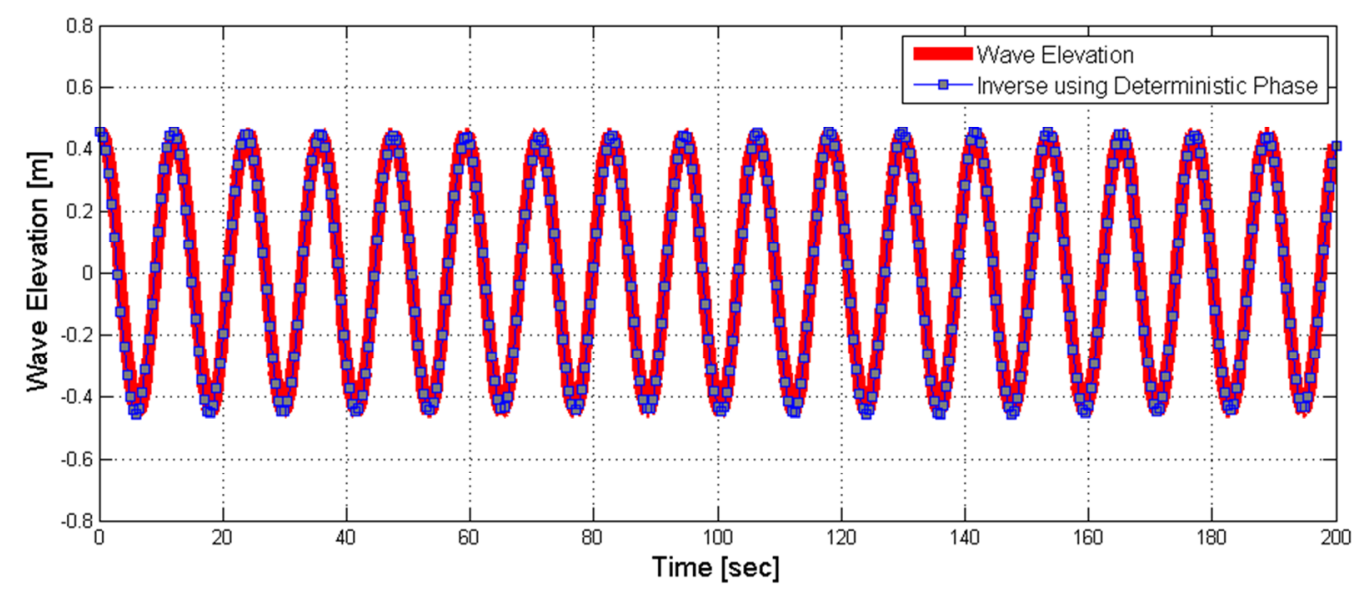

Fig. 6 Comparison between theoretical regular wave and its inversion using deterministic phase as eq. (12)

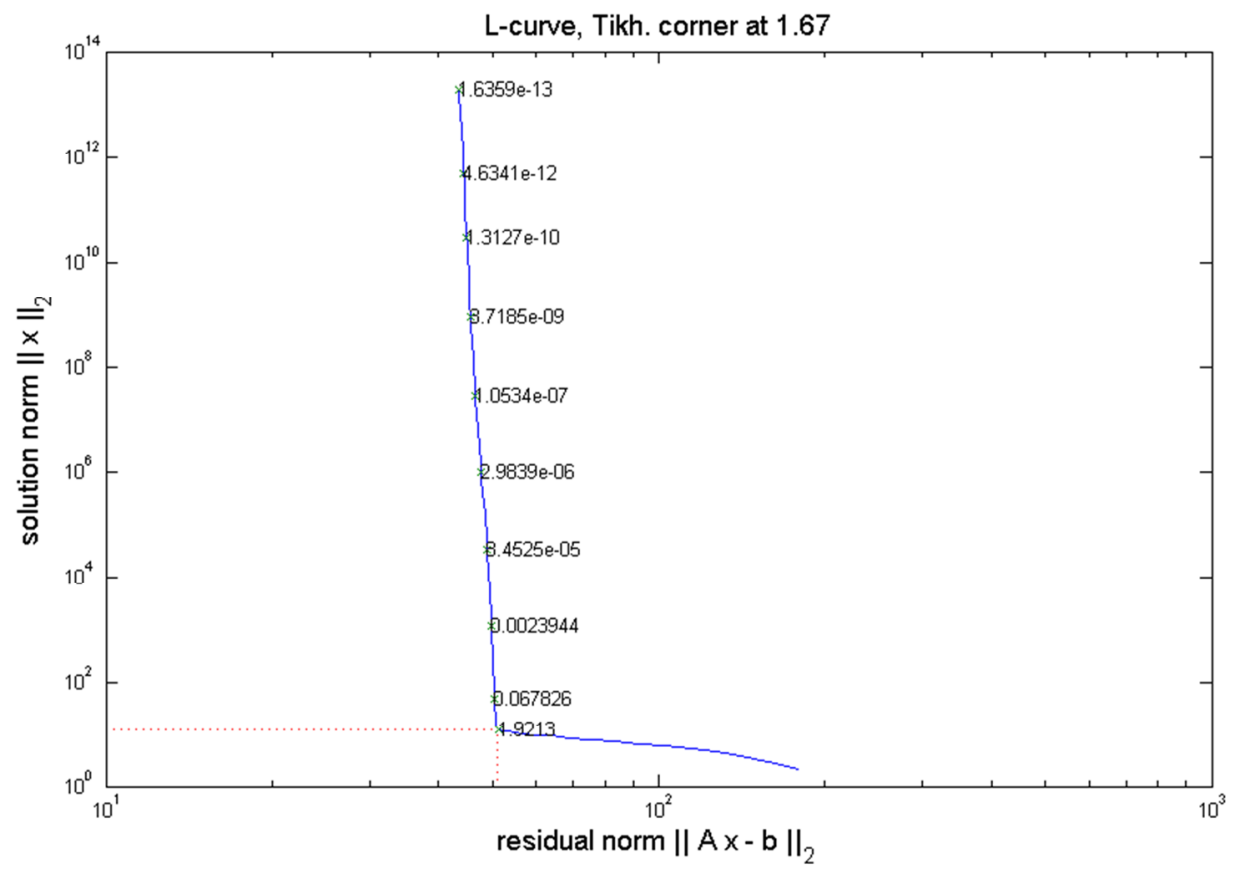

Fig. 7 Result of L-Curve for irregular wave

5.2 Irregular wave prediction using calculated deterministic phase

Examples of predictions on irregular waves involve the following procedure:

a. Generate a JONSWAP spectrum under the condition of $H_{S}=10 \mathrm{~m}$ and $T_{Z}=10 \mathrm{~s}$ from $0 \mathrm{~s}$ to $200 \mathrm{~s}$ (red line in Figs. 8 to 11).

b. Using the above condition, generate an irregular wave, and to compare the prediction results, generate reference irregular waves at points $0 \mathrm{~m}, 200 \mathrm{~m}, 400 \mathrm{~m}$, $600 \mathrm{~m}$, and $800 \mathrm{~m}$.

c. Calculate the deterministic phase using the reference irregular wave from $0 \mathrm{~s}$ to $140 \mathrm{~s}$ at $0 \mathrm{~m}$ position (red line in Figs. 8 to 11 ).

d. Use the calculated deterministic phase to predict waves at points $200 \mathrm{~m}, 400 \mathrm{~m}$, $600 \mathrm{~m}$, and $800 \mathrm{~m}$, and compare them to the reference irregular waves at the same points. 


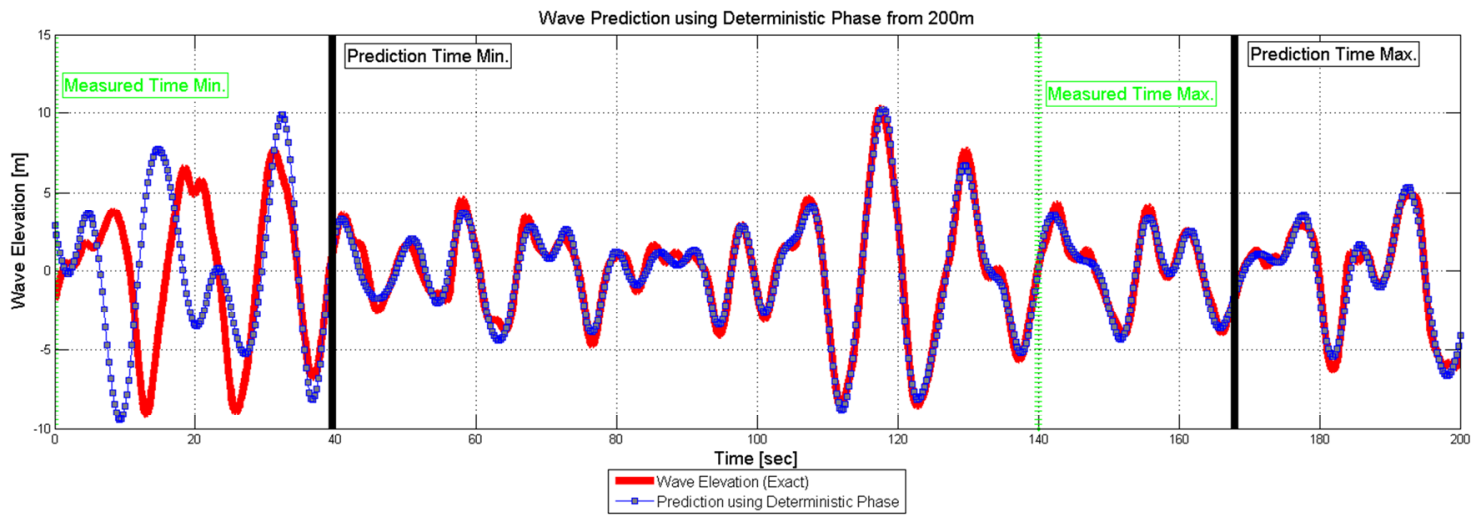

Fig. 8 Comparison between prediction wave and exact wave elevation at a distance of $200 \mathrm{~m}$

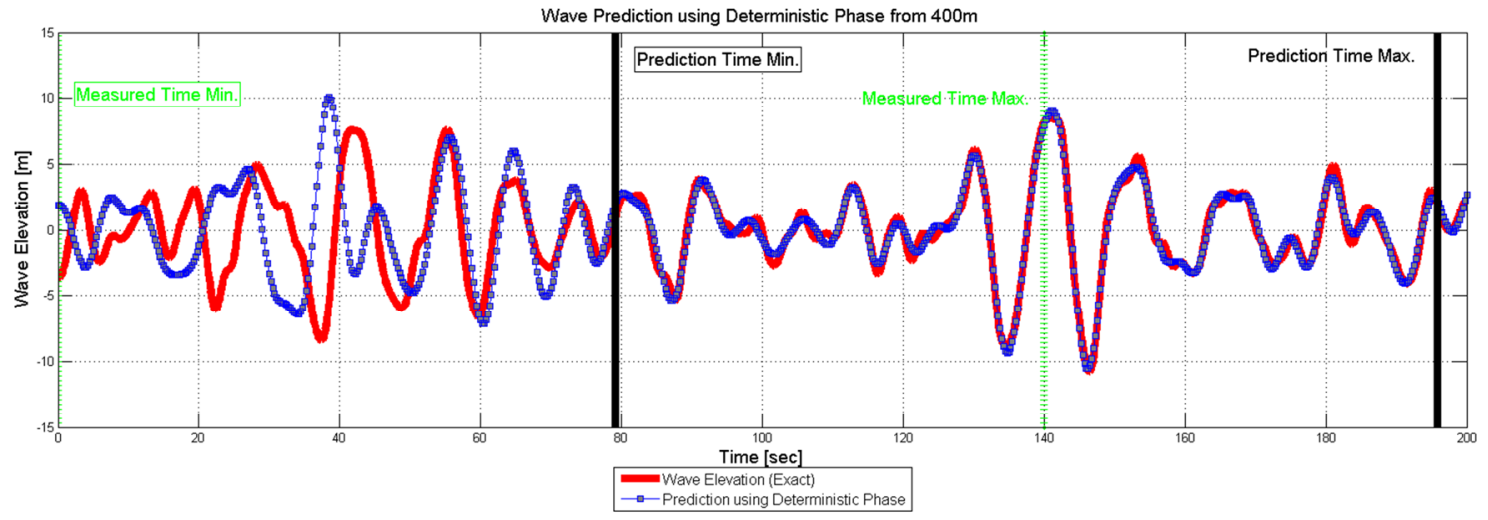

Fig. 9 Comparison between prediction wave and exact wave elevation at a distance of $400 \mathrm{~m}$

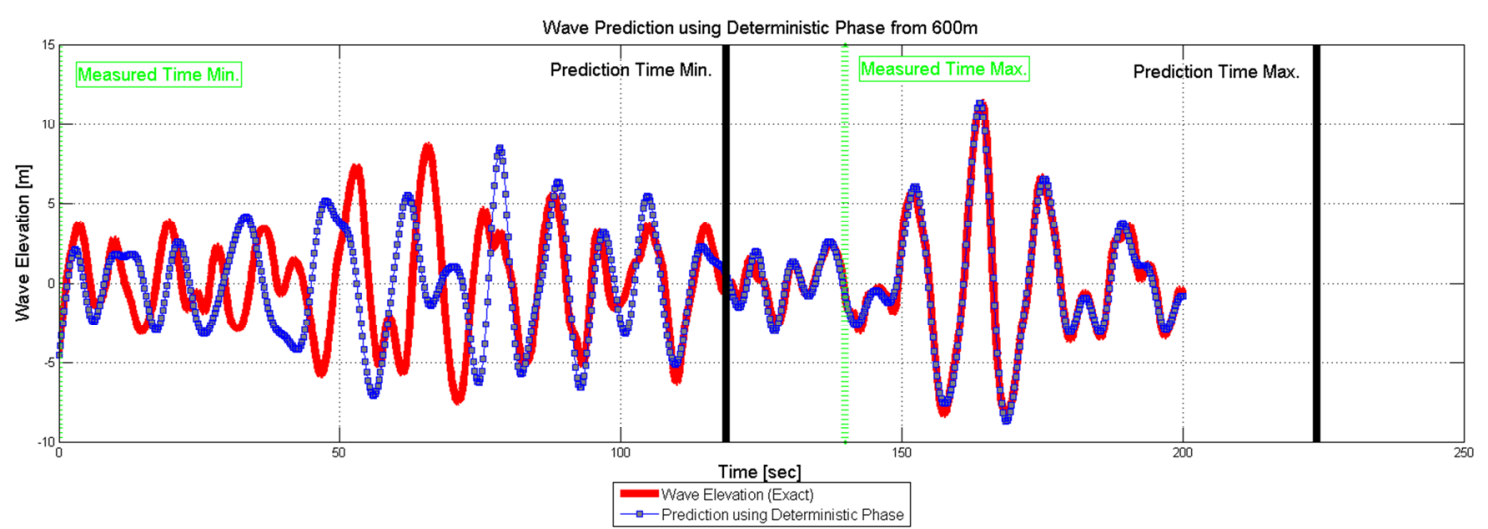

Fig. 10 Comparison between prediction wave and exact wave elevation at a distance of $600 \mathrm{~m}$

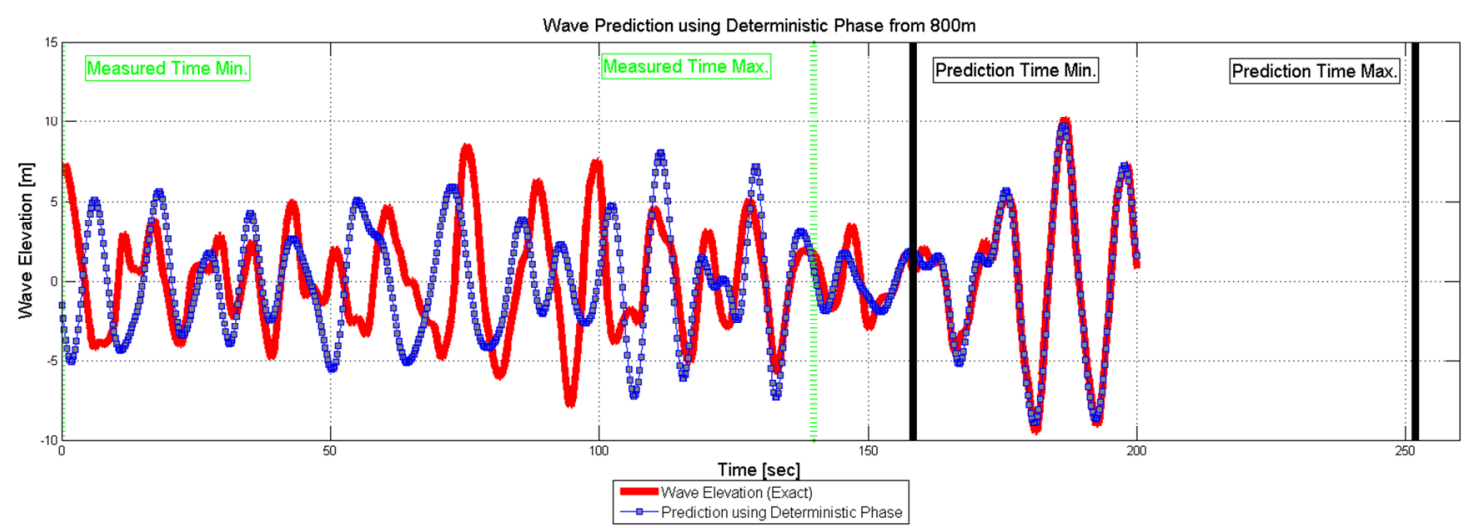

Fig. 11 Comparison between prediction wave and exact wave elevation at a distance of $800 \mathrm{~m}$ 


\section{Conclusion}

The deterministic phase matrix $P$ calculated by the inverse method can be used in the inversed irregular wave calculation by using the matrices $T$ and $A$, obtained previously from eq. (12), and the calculated inverse irregular wave can be directly compared with the theoretical irregular wave. Figs 8 to 11 compare the theoretical irregular wave and the predicted irregular wave using the deterministic phase calculated by the inverse method. The red dashed line is the theoretical irregular wave, and the blue dotted line is the predicted irregular wave.

Moreover, Figs 8 to 11 show the prediction results of the irregular waves. The black vertical line represents the minimum - maximum times at which prediction is possible and is calculated by using Fig. 4. To calculate the predictable times from Fig. 4, minimum and maximum frequency values must be set. Values of $75 \%$ of the minimum and maximum frequencies were used in this study.

The region between the black vertical lines is the predictable region, and it can be seen that at each position from 200 to $800 \mathrm{~m}$, the predictions match the exact irregular waves.

The regularization method proposed for use in irregular wave predictions was introduced to solve the ill-conditioned problem in calculating inverse matrices, and the Lcurve method was introduced to minimize errors in Fig. 7.

In particular, the L-curve method allowed us to determine an optimum $\alpha$ value, and as this value increases, the solution tended to stabilize, but if it is too large, it is difficult to obtain an exact solution. Therefore, controlling the $\alpha$ value is important in stabilizing the solution and finding an exact solution. It is very important to find the optimum L-curve corner.

\section{ACKNOWLEDGEMENT}

This work was supported by the National Research Foundation of Korea (NRF) grant funded by the Korean government (MSIP) through GCRC-SOP (No. 2011-0030013). This research was supported by a grant by the LNG Plant R\&D Center which belongs to the Plant Research Program funded by the Ministry of Land, Infrastructure and Transport (MOLIT) of the Korean government. This work was also funded by the Korea Meteorological Administration Research and Development Program under Grant KMIPA 2014-22010. The paper is based on the $\mathrm{PhD}$ thesis.

\section{REFERENCES}

[1] Young I. R., and Rosenthal W., A Three-Dimensional Analysis of Marine Radar Images for the Determination of Ocean Wave Directionality and Surface Currents, Journal of geophysical Research, 90, no. C1, 1049-1059, 1985. DOI:10.1029/JC090iC01p01049

[2] Atanassov V., Rosenthal W., and Ziemer F., Removal of Ambiguity of Two-Dimensional Power Spectra Obtained by Processing Ship Radar Images of Ocean Waves, Journal of geophysical Research, 90, no. C1, 1061-1067, 1985. DOI:10.1029/JC090iC01p01061

[3] Ziemer, F. and Günther, H., A System to Monitor Ocean Wave Fields. Proc. 2nd Int. Conf. On Air-Sea Interaction and Meteorology and Oceanography of the Coastal Zone. Lisboa, September 22-27, 1994. DOI:10.1109/oceans.1994.364010

[4] Nieto J., Rodriguez G., Hessner K., Izquierdo P., Inversion of Marine Radar Images for Surface Wave Analysis. Journal of Atmospheric and Oceanic Technology, 21, no. 8, 1291-1300, 2004. DOI:10.1175/1520-0426(2004)021<1291:IOMRIF>2.0.CO;2

[5] Blondel E., and Naaijen, P., Reconstruction and Prediction of Short-Crested Seas Based on the Application of a 3d-FFT on Synthetic Waves. Part 2: Prediction, International Conference on Ocean, Offshore and Arctic Engineering, 5, 43-53, 2012.

[6] Blondel E., and Naaijen, P., Deterministic Prediction of Ocean Waves Based on X-Band Radar Measurements, 13emes Journees de l'Hydrodynamique, 2012 
[7] Naaijen P., and Hujismans R., Real Time Wave Forecasting for Real Time Ship Motion Predictions, International Conference on Offshore Mechanics and Arctic Engineering, 4, 607-614, 2008. DOI:10.1115/omae2008-57804

[8] Clauss G., Kosleck S., and Testa D., Critical Situations of Vessel Operations in Short Crested Seas Forecast and Decision Support System, International Conference on Offshore Mechanics and Arctic Engineering, 4, 319-332, 2009. DOI:10.1115/omae2009-79482

[9] Naaijen P. van Dijk R. T., Hujismans R. H. M., and El-Mouhandiz A. A., Real time estimation of ship motions in short crested seas, International Conference on Offshore Mechanics and Arctic Engineering, 4, 243-255, 2009. DOI:10.1115/omae2009-79366

[10] Wu G., Direct Simulation and Deterministic Prediction of Large-scale Nonlinear Ocean Wave-field, PhD Thesis, Massachusetts Institute of Technology, 2004.

[11] Blondel, E., Reconstruction et prévision déterministe de houle à partir de données mesurées, PhD Thesis, Ecole Centrale de Nantes, 2009.

[12] Kosleck S., Prediction of Wave-Structure Interaction by Advanced Wave Field Forecast, PhD Thesis, Technische Universität Berlin, 2013.

[13] Naaijen P., and Hujismans R., Real Time Prediction of Second Order Wave Drift Forces for Wave Force Feed Forward in DP, International Conference on Ocean, Offshore and Arctic Engineering, 4, 357-364, 2010. DOI:10.1115/OMAE2010-20618

[14] Kwon S.H., Kim C.H., and Jang T.S., An Identification of Wave Propagation Based on a Single-Point Measurement, Ocean Engineering, 34, no. 10, 1405-1412, 2007. DOI:10.1016/j.oceaneng.2006.10.008

[15] Blondel E., Bonnefoy F., and Ferrant P., Deterministic Non-Linear Wave Prediction using Probe Data, Ocean Engineering, 37, no. 10, 913-926, 2010. DOI:10.1016/j.oceaneng.2010.03.002

[16] Zhang, J., Yang, J., Wen, J., Prislin, I., and Hong, K., Deterministic wave model for short crested ocean waves - Part I: Theory and numerical scheme, App. Ocean Res., 21, 167-188, 1999. DOI:10.1016/S0141-1187(99)00011-5

[17] Zhang, J., Prislin, I., Yang, J., and Wen, J., Deterministic wave model for short crested ocean waves Part II: Comparison with laboratory and field measurements, App. Ocean Res., 21, 189-206, 1999. DOI:10.1016/S0141-1187(99)00012-7

[18] Belmont M.R., Horwood J.M.K, and Thurley R.W.F., Baker J., Filters for linear sea-wave prediction, Ocean Engineering, 33, 2332-2351, 2006. DOI:10.1016/j.oceaneng.2005.11.011

[19] Li G., Weiss G., Mueller M., Townley S., and Belmont M.R., Wave energy converter control by wave prediction and dynamic programming, Renewable Energy, 28, 392-403, 2012. DOI:10.1016/j.renene.2012.05.003

[20] Kim C.H., Nonlinear Waves and Offshore Structures. World Scientific, 2008. DOI:10.1142/4906

[21] Janssen T. T., Van Dongeren A. R., and Kuiper C., Phase Resolving Analysis of Multidirectional Wave Trains, Proceedings of Ocean Wave Measurement and Analysis, 377-387, 2001.

[22] Welch, D., The Use of Fase Fourier Transform for the Estimation of Power Spectra: A Method Based on Time Averaging Over Short, Modified Periodograms, 15, no. 2, 70-73, 1967.

[23] Johnston P. R., and Gulrajani R. M., Selecting the Corner in the L-Curve Approach to Tikhonov Regularization, IEEE Transactions on Biomedical Engineering, 47, no. 9, 1293-1296, 2000. DOI:10.1109/10.867966

Submitted: $\quad 23.11 .2015$

Accepted: $\quad 20.9 .2016$
Young Jun Yang

ybihong@pusan.ac.kr

Global Core Research Center for Ship and Offshore Structure \#209, Pusan National University, Geumjeong-gu, Busan, 46241, Republic of Korea (South Korea)

Sun Hong Kwon shkwon@pusan.ac.kr Department of Naval Architecture and Ocean Engineering, Pusan National University, Geumjeong-gu, Busan, 46241, Republic of Korea (South Korea) 